\title{
MYELOMA - THE INTEGRAL ROLE PLAYED BY THE PROFESSIONAL NURSE
}

\author{
Lucille Wood, Peter Jacobs
}

\section{INTRODUCTION}

Many of the clinical features and biochemical abnormalities of myeloma were described more than 100 years ago, and in the late stages of the disease there should be little difficulty in making the diagnosis. Patients typically have severe bone pain associated with lytic lesions in the skeleton, while anaemia in the presence ff a high erythrocyte sedimentation rate, progressive renal dysfunction and serious infections combine to produce a distinctive constellation of symptoms and signs. Despite this, a number of cases are still missed because many individual practitioners have limited experience, having seen few examples during their careers or through not maintaining a sufficiently high index of clinical suspicion to recognise the telltale findings.

Understandably, this deficiency is transmitted to nursing and paramedical professionals since less attention is paid in their training to the diagnosis of this disease and consequently the role of these colleagues in management is significantly under-utilised.

There are sound reasons for doctors and nurses alike to remain alert to this diagnostic possibility because, once established, many of the initial abnormalities can be relatively easily versed, thereby improving the quality of life. Furthermore, using the appropriate combinations of chemotherapy and radiotherapy, survival can be extended from 7 to approximately 30 months. While these achievements are notable, they leave much to be desired when the outlook, especially in younger patients, is compared to other haematologic malignancies, such as Hodgkin's disease, where cure has become the aim of treatment. Not surprisingly, therefore, much research and development is in progress to define the role of newer treatment options that include fractionated half-body irradiation, biologic immune response modulation with interferon and, particularly, high doses of cytotoxic drugs in combination with whole body radiotherapy as a prelude to bone marrow transplantation.

To bring perspective to current management and to emphasize the role of the professional nurse, the more important clinical and laboratory features are briefly reviewed.

\section{DIAGNOSIS}

The accumulation of plasma cells is the

\section{Abstract}

Myeloma is a malignancy of plasma cells which are terminally differentiated B-lymphocytes. The diagnosis may be made incidentally at routine blood resting, when an abnormality is found in the plasma proteins on electrophoresis. More usually the patients are symptomatic, with bone pain, anaemia, evidence of renal failure, or the metabolic abnormalities associated with increased plasma calcium and urate levels. Effective treatment will extend survival from 7 to approximately 30 months and at the same time improve the quality of life. Treatment is multidisciplinary, prominently involves the professional nurse and may arbitrarily be divided into two stages. Firstly, reversible lesions, such as dehydration and plasma hyperviscosity must be corrected. hypercalcaemia and hyperuricaemia improved and, if necessary, renal dialysis undertaken. Secondly, but of equal importance, is the need for specific therapy to be directed against the tumour itself, and both cytotoxic agents and irradiation have an important role to play. More recently, newer approaches have included high dose chemotherapy and bone marrow transplantation.

end stage of uncontrolled overgrowth or clonal expansion of B-lymphocytes. The tumour cells have a distinctive morphology, with an eccentric nucleus and often abundant cytoplasm containing a high concentration of protein, leading to intense blue staining when the

Romanowsky dyes are used (Figure 1). Normally, a wide range of antibodies is produced by cells of the immune system, thereby providing protection from invading organisms. However, when malignant change arises in a single cell and leads to myeloma, large amounts of a particular immunoglobulin are

synthesized, in contrast to the situation in health. It is this change that is recognised in the protein electrophoretic pattern

(Figure 2). Yet an alternative feature is the presence of light-chains from the abnormal immunoglobulin, known as Bence Jones protein, in the urine. The combination of an abnormal concentration of plasma cells, as for example in the bone marrow, combined with the presence of a paraprotein peak in the plasma are the two cardinal features for diagnosis (Table 1).

A number of consequences follow disease progression, such as the development of lytic lesions in bone, bone marrow failure with development of anaemia and thrombocytopenia, marked increase in whole blood viscosity with disturbances in mental function and a variety of metabolic changes, of which elevation in plasma calcium and uric acid levels are the most typical. A word of warning is appropriate since many of these findings can occur in other diseases, with metastatic cancer affecting the skeleton, hypercalcaemia in tuberculosis, and elevated uric acid in primary gout. It is therefore important that as many of the individual features as possible are combined to make a positive diagnosis of myeloma.

\begin{tabular}{|c|}
\hline $\begin{array}{l}\text { TABLE 1 } \\
\text { Diagnostic Criteria in Myeloma } \\
\text { This malignancy is most reliably diagnosed when } \\
\text { the accepted criteria are observed. It is noteworthy } \\
\text { that a number of the associated features occur in } \\
\text { other diseases and care should be exercised before } \\
\text { attributing them to this immunoproliferative dis- } \\
\text { order. A confident diagnosis will be supported by } \\
\text { any two major criteria, one major and two minor } \\
\text { criteria, or different combinations thereof. }\end{array}$ \\
\hline $\begin{array}{l}\text { MAJOR CRITERIA } \\
\text { 1. Plasmacytoma on tissue biopsy } \\
\text { 2. Bone marrow plasmacytosis }>30 \% \\
\text { 3. Monoclonal immunoglobulin peak } \\
-\lg (;>35 \mathrm{~g} / \mathrm{L} \\
-\operatorname{IgA}>20 \mathrm{~g} / \mathrm{L} \\
- \text { Urinary light chain excretion }>1 \mathrm{~g} / 24 \text { hours }\end{array}$ \\
\hline $\begin{array}{l}\text { MINOR CRITERIA } \\
\text { 1. Bone marrow plasmacytosis between } 10 \text { and } 30 \% \\
\text { 2. Monoclonal immunoglobulin peak less than } \\
\text { for major criteria } \\
\text { 3. Lytic bone lesions } \\
\text { 4. Kesidual normal or polyclonal immunoglobu- } \\
\text { Ilns reduced } \\
-\operatorname{lgM}<, 5 \mathrm{~g} / \mathrm{L} \text {, } \\
-\mathrm{IgA}<1 \mathrm{~g} / \mathrm{L} \\
-\mathrm{IgG}<6 \mathrm{~g} / \mathrm{L}\end{array}$ \\
\hline
\end{tabular}

DISEASE EXTENT OR STAGING

Since response to therapy and survival are both correlated with the amount of tumour present in the patient, it is of practical importance to separate individuals into those with limited, or stage I, disease from 


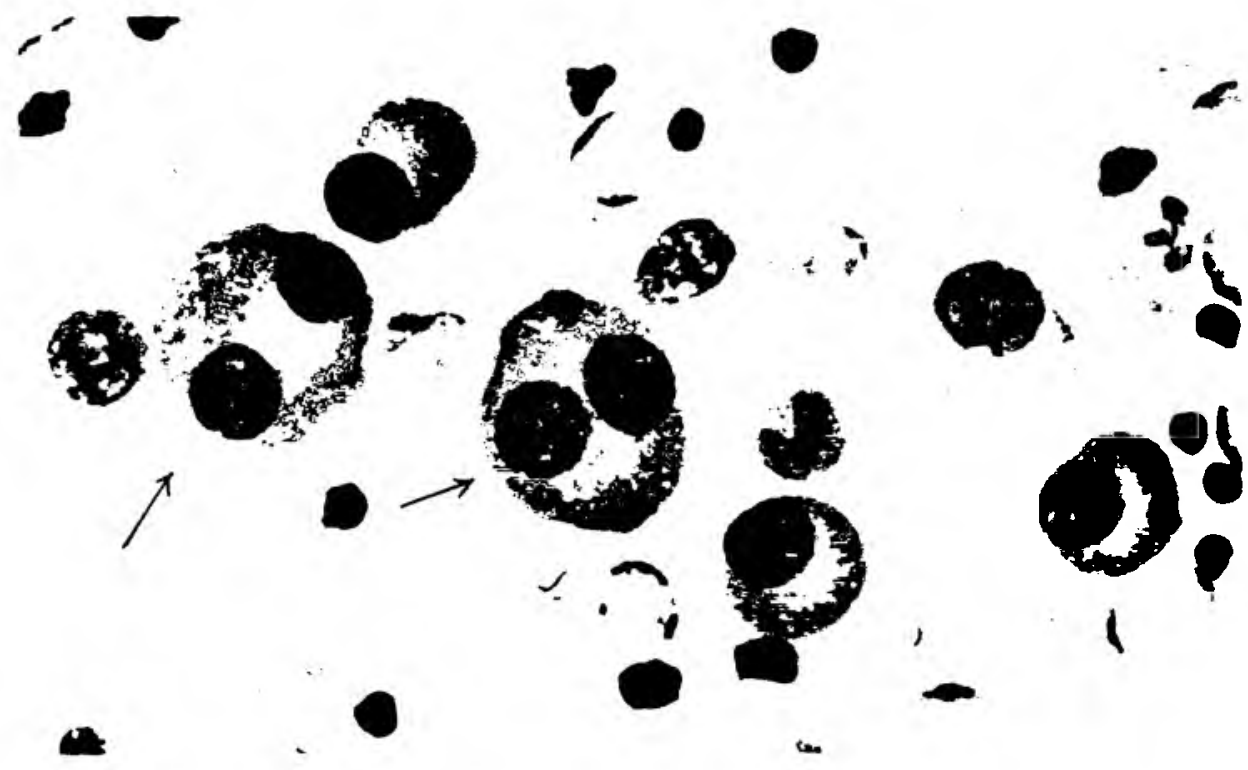

Fig. 1. THE MARROW IN MYELOMA There is a striking increase in plasma cells, with their eccentric nucleus and abundant amounts of cytoplasm. In addition, obviously malignant forms are present, and these are of large size and grotesquely distorted nuclei (arrow).

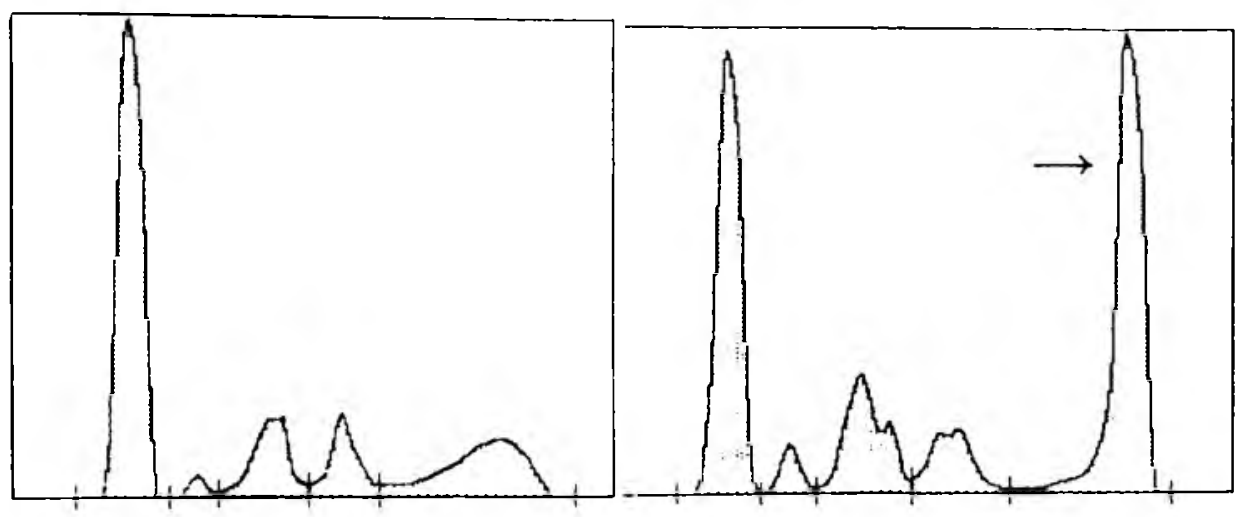

Fig. 2. PLASMA ELECTROPHORESIS In contrast to the normal pattern, shown in the left-hand panel, the large amount of abnormal immunoglobulin, which is known as paraprotein, is strikingly evident in the right-hand panel (arrow).

those with high bulk, or stage III, and to recognise that between these two extremes exists a group defined by their being unable to fulfill the above criteria (Table 2).

\section{PROGNOSTIC FACTORS}

Apart from the need to establish an accurate diagnosis and then to define the extent of disease present in the patient, other features have been recognised that have an influence on outcome of treatment; these variables are called predictive or prognostic factors. In general terms, the younger the patient with myeloma the greater the likelihood of high bulk disease, extensive skeletal damage and associated metabolic disorders or renal failure. Furthermore, marked elevations in serum lactic dehydrogenase level and a particular serum protein known as beta-2microglobulin have significant correlation with poor outcome. In the laboratory there are other measurements useful in recognising poor risk patients that include cytogenetic abnormalities and the fraction of dividing cells in the tumour, which can be determined with radioactive isotopes; this is known as the labelling index.

\section{CLINICAL FEATURES}

At the one extreme are localised areas of tumour, known as plasmacytomas, and at the other is active widespread disease that gives rise to the characteristic symptoms and signs. An intermediate pattern may be recognised in which the neoplastic process advances slowly, and these individuals are said to have the indolent or smouldering form. At the benign end of this indolent variant are some individuals who have only an abnormal immunoglobulin pattern on serum electrophoresis, which remains stable for years. The latter entity is described as monoclonal gammopathy of undetermined significance, and while some of these patients progress to myeloma, others die without undue change in the protein pattern taking place.

The most striking symptom of active or advancing disease is that of bone pain. Typically, this occurs in the axial skeleton
TABLE 2

Staging in Myeloma

The amount of disease bulk has a direct bearing an response to therapy and survival. It follows that al patients should be classified according to currently acceptable international criteria.

Stages are subclassified as $A$ or $B$ according to renal function:

$\mathrm{A}=$ creatinine $<2 \mathrm{mg} / 100 \mathrm{~mL}$ or $\mathrm{BUN}<30 \mathrm{mg} / 100$ mL;

$B=$ creatinine $>2 \mathrm{mg} / 100 \mathrm{~mL}$ or $\mathrm{BUN}>30 \mathrm{mg} / 100$ m L

* Corrected calcium $(\mathrm{mg} / \mathrm{dL})=$ serum calcium $(\mathrm{mg} / \mathrm{dL})$ serum albumin $(\mathrm{g} / \mathrm{dL})+4,0$

\section{$\begin{array}{ll} & \text { All of the following criteria: } \\ \text { STAGE I } & - \text { Haemoglobin }>100 \mathrm{~g} / \mathrm{L}\end{array}$}

Low myeloma cell mass $<0,6 x$ $10^{12}$ cells $/ \mathrm{m}$

- Serum calcium (corrected) $<12,0$ $\mathrm{mg} / \mathrm{dL}$ *

- $X$-rays reveal normal bone structure or solitary lesion

$-M$ component production values IgG $<50$ g/ L

$\lg \mathbf{A}<\mathbf{g} / \mathbf{L}$ $\mathrm{g} / 24 \mathrm{hr}$

Urine light chain excretion $<\mathbf{4 0}$

STAGE II Intermediate myeloma cell mass 0,6 to $1,2 \times 10^{12} \mathrm{cells} / \mathrm{m}^{2}$

Criteria fit neither Stage I nor Stage III

High myeloma cells mass $>1,2 \mathrm{x}$ $10^{12}$ cells $/ \mathrm{m}^{2}$

Any of the following criteria

- Haemoglobin $<85 \mathrm{~g} / \mathrm{L}$

STAGE III - Serum calcium (corrected) $>12,0$ g/dI,

- Advanced lytic bone lesions

$-\mathbf{M}$ component production values $\operatorname{IgG}>70 \mathrm{~g} / \mathrm{L}$

$\mathrm{IgA}>50 \mathrm{~g} / \mathrm{dL}$

Urine light chain excretion $>$ $120 \mathrm{~g} / \mathrm{L} / 24 \mathrm{hr}$

and many patients complain of it being worse at night. The underlying abnormalities are usually found to be lytic lesions, sometimes with a pathological fracture through the involved area, although there may be diffuse loss of bone or osteoporosis resulting in compression fracture of the vertebral bodies.

Renal dysfunction may occur for a number of reasons, of which the most important is that of dehydration. Associated disturbances are attributable to increases in serum calcium and uric acid levels. The kidney may be directly infiltrated with plasma cells or deposition of a material known as amyloid. A particularly interesting disturbance arises when part of the immunoglobulin molecule, the light-chains or Bence Jones proteins, is deposited in the tubules, and this occurs usually when these fragments are excreted in the urine. It is important to appreciate that many of the factors contributing to abnormalities in renal biochemistry can be reversed and the older concept that all such patients of necessity have a poor outlook is no longer acceptable.

Haematologic abnormalities typically are the presence of mild to moderate degrees of anaemia, with normochromic normocytic red cells. The usual cause is bone marrow infiltration by the tumour, but there may be associated shortening of red cell survival or haemolysis. 
Thrombocytopenia may occur, leading to haemorrhage from the gastrointestinal tract, and this may be aggravated by impaired platelet function in patients with severe degrees of uremia and increase in the blood urea level. The marked decrease in normal gammaglobulin leads to immunologic incompetence, with bacterial infections that may be recurrent and most frequently involve the lungs.

Neurologic complications follow vertebral collapse or extradural spinal cord deposits of the tumour cells. Less frequently, peripheral neuropathy may be associated with amyloid and on rare occasions solitary plasmacytomas are found within the brain substance.

The raised plasma protein levels give rise to hyperviscosity and accounts for the marked increase in erythrocyte sedimentation rate, compounds the degree of renal dysfunction and typically leads to severe degrees of mental confusion.

\section{URSING ASPECTS}

There are a number of areas where the academically orientated nursing professional has an important role to play. It is necessary to strongly emphasize that in the contemporary context there is emerging a recognition that contributions from these colleagues are seriously and significantly under-utilised. Strenuous efforts are being made, particularly in the United States of America, to harness this resource by developing career structures or ladders that will bring equivalence at senior faculty level to the more traditional routes for promotion into administration or education. This is the concept of the Clinical Nurse Specialist, and it is not only appropriate but important and somewhat overdue that similar consideration and efforts be made by local statutory bodies in this country.

More specifically, and in the outpatient clinic, strong psychological support is derived by integrating medical and nursing services into a multidisciplinary health care team. Not infrequently the medical profession are inadequately sensitive to patient anxieties and many questions about treatment details, side effects and even survival are directed to the sister involved with moment-to-moment care. These concerns range from such simple procedures as red cell transfusions to administration of the often highly complex chemotherapy programmes and, more recently, to irradiation or even bone marrow transplantation. An ideal opportunity to generally reassure the patient or organise referral to the social worker on issues that range from loss of earning power to care for the family arise when blood or urine samples are being collected. As part of the modern treatment programmes, blood transfusions may be replaced by the administration of recombinant human erythropoietin. Since these protocols are carried out in the outpatient clinics and involve regular data collection it is entirely appropriate that nursing colleagues are equal coinvestigators in such developmental work and share the recognition that is derived from presentation at meetings and publication of trial results.

At least in our department there is yet a further area where the Professional Nurse plays an important role and is delegated direct responsibility for management decisions. This is in the use of therapeutic apheresis to reverse hyperviscosity by

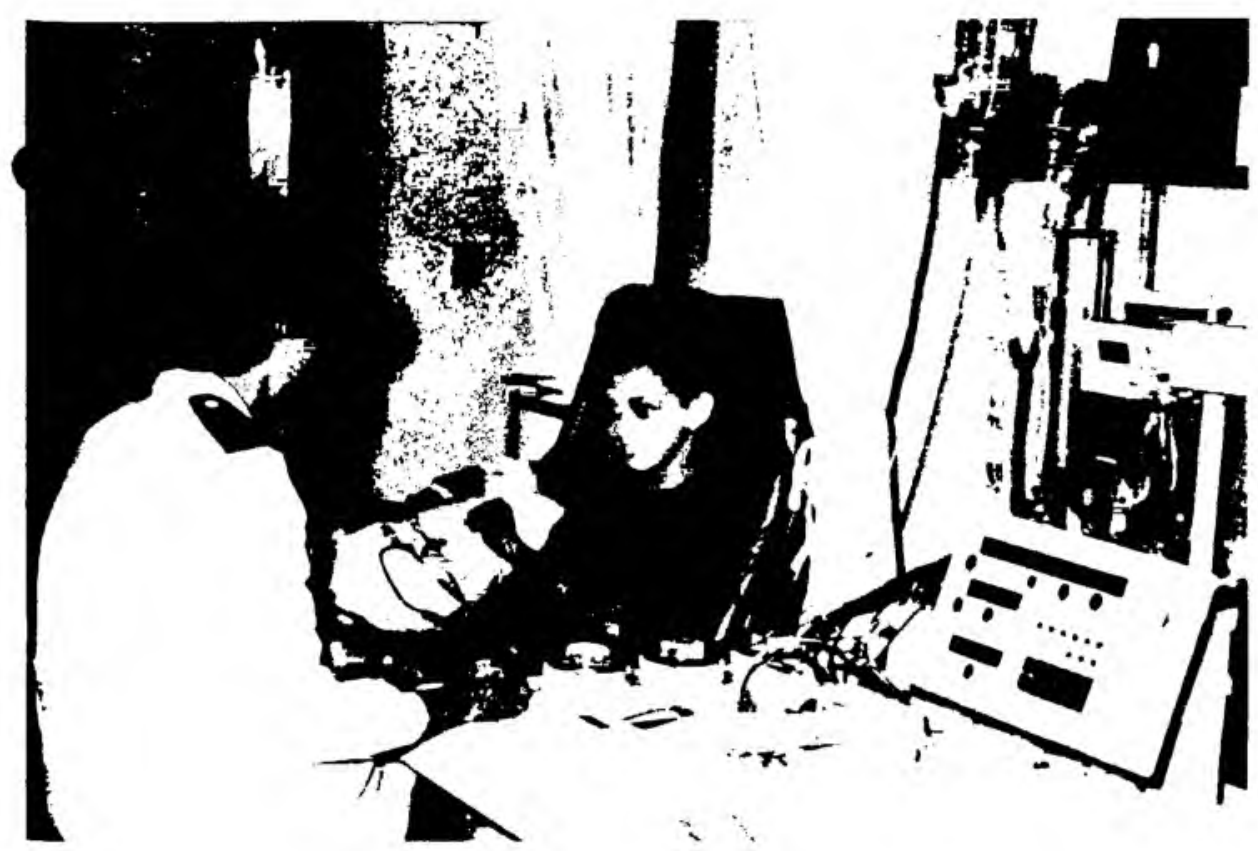

Fig. 3. BLOOD FRACTION SEPARATOR

This instrument - the Cobe 2997 - exemplifies the sophisticated technology that has made it possible to remove the patient's plasma with its high concentration of abnormal immunoglobulin and to replace this with an electrolyte solution containing albumin. This methodology is highly efficient and will return plasma and whole blood viscosity to normal, with objective improvement in cerebral function often strikingly evident within 12 hours.

means of continuous-flow blood fraction separation (Figure 3), and this would exemplify academic expertise commensurate with the specialist or senior specialist in the medical hierarchy, corresponding to the Clinical Nurse Specialist referred to above. In this technique the sister establishes a closed vein-to-vein circuit that includes the centrifuge in the system. The circulating blood is split into its fractions, based on density, and the red cells simply return to the patient, while the abnormal plasma containing the paraprotein is discarded. Shortly before the patient receives his own red cells back, an electrolyte solution containing albumin is mixed so that approximately normal blood viscosity is re-established. The symptoms attributable to poor perfusion of the microcirculation in the patient may result in striking improvement in cerebral function, sometimes whilst on the machine, but invariably within the first 24 hours.

Another area of responsibility is nursing management of the hospitalised patient. Here, wide ranging responsibilities includes monitoring of fluid balance during treatment of hypercalcaemia with forced diuresis, requiring an awareness of the need to replace potassium and magnesium, regular charting of responses in plasma calcium level and ensuring that neither dehydration nor fluid overload occur

There are additional important aspects to nursing the patient with bone pain due to lytic lesions, and an understanding of the role played by local radiotherapy and the judicious use of analgesia go a long way towards ensuring patient comfort. Unlike the misconception that nurses exist simply to carry out medical instructions, we strongly hold the viewpoint that optimum care of patients, those with myeloma providing an excellent illustration, requires a multidisciplinary health care team. In this particular context it is difficult to overestimate the role of the academically orientated and trained nurse who has kept her expertise up-todate. We consider these individuals to be specialists in their own right and it is appropriate that their responsibilities extend to the administration and monitoring of cytotoxic drug therapy. There is a need to have a thorough knowledge of the protocols currently in use, a familiarity with the effects and complications of cytotoxic drugs and be able to supervise the administration of an optimal anti-emetic regimen that will markedly contribute to patient comfort and safety.

As though these challenges were not enough, the role of colleagues who remain at the forefront of nursing and medical advances should extend to the guidance of junior medical and nursing staff, particularly registrars rotating through departments, whose knowledge of complex management schedules is often woefully inadequate. There is a need to interact confidently with clinical and laboratory specialists in haematology and also to provide the effective co-ordination of support that may extend to the provision 
of allogeneic platelets, therapeutic plasma exchange, haemodialysis or intubation and assisted ventilation. Superimposed upon these aspects of comprehensive management is the additional need to be vigilant for the onset of serious and often life-threatening infections, shock, collapse in blood pressure, deterioration in renal failure and development of disseminated intravascular coagulation.

It is our cumulative experience that optimum patient care is most efficient and cost-effectively achieved where a multidisciplinary group exists that comfortably accommodates the academic aspirations of the professional nurse as well as paramedical professionals. We do not consider it any longer appropriate to view nursing colleagues, most specifically the academically-orientated professional nurse, as the handmaiden of the physician!

\section{CONVENTIONAL THERAPY}

Currently, most regimens would employ an alkylating agent in the form of melphalan in combination with prednisone. This simple approach remains the cornerstone aganst which other treatment options are measured. In general terms, the addition of further drugs has done little to improve the median survival, which has been extended from 7 to about 30 months with the twodrug programme.

Response in patients can be monitored by improvement in the stage and most importantly in the performance status or Karnofsky rating. However, a number of individuals will be completely resistant or refractory to chemotherapy, and such a situation is associated with short survival. A further alternative is that relapse may occur after initial response and in these circumstances the tumour cells may change their characteristics and are said to have undergone escape by mutation. In both of these situations the treatment options are now more restricted and involve secondline drugs, generally with less satisfactory disease control or survival.

Our own experience, based on the undoubted benefits of irradiation to local lesions, has been to treat larger parts of the body with radiotherapy. The upper or the lower half could be treated sequentially, and this has been shown to be effective for patients who have relapsed on chemotherapy. It is currently not clear whether such systemic radiotherapy will offer any advantages over conventional chemotherapy.

\section{COMPLICATIONS OF \\ CONVENTIONAL THERAPY}

Apart from the failure to respond or relapse in some individuals, the use of either radiotherapy or chemotherapy is associated with significant side effects. Thus, worsening of bone marrow function may lead to degrees of neutropenia or thrombocytopenia, restricting the amount of treatment that can be given and thereby compromising tumour kill. In addition, there may be adverse effects of the treatment itself, including the emergence of preleukaemia or acute nonlymphoblastic leukaemia.

\section{DEVELOPMENTAL, APPROACHES TO MANAGEMENT}

Because of the difficulties with chemotherapy, many studies have been undertaken exploring alternative options.

Two rather different approaches are currently the subject of investigation. In the first of these is the use of recombinant human alpha interferon. This interesting molecule is produced by viruses and one of the effects noted is suppression of cell division, including the bone marrow. Based on that observation, such biologic immune response modulation has been attempted in patients with myeloma. Initially, studies were carried out on patients who had late or end-stage disease, with poor results. More recently, it has been suggested that interferon used early, either alone or preferably in combination with one of the established drug regimens, may achieve better results. Early reports are encouraging, but have not as yet been confirmed

The other alternative is the use of escalating doses of single agent chemotherapy, usually in the form of melphalan, that would otherwise be lethal because of the way in which it produces irreversible bone marrow damage. With the wider availability of bone marrow transplantation, it has become practical to use the very large quantities of the alkylating agent, often in combination with radiotherapy, and then to rescue the patient from the myelotoxicity with either allogeneic or autologous bone marrow transplantation. This treatment option is of undoubted benefit, but is limited somewhat by the scarcity of transplant centres and the fact that many of the patients are elderly so that the conditioning regimens needed to eradicate the disease are associated with substantial morbidity and mortality. Nevertheless, particularly in the younger patients, this remains the single most interesting addition to the therapeutic options for treating patients with myeloma.

As with nursing in general, each of these programmes is critically dependent upon direct involvement of our professional colleagues. In biologic immune response modulation is the need to administer the agent, monitor the influenza-like syndromes that occur, and constantly be on the look-out for serious central nervous system signs and symptoms. In bone marrow transplantation, nurses play a role in the theatre, collecting marrow and subsequently caring for the immunocompromised patient in the protected environment. The latter units, whilst having sisters-in-charge, are best supervised by an academic colleague corresponding to the Clinical Nurse Specialist in the American system.

\section{QUALITY OF LIFE}

There is an important ethical and moral responsibility to fully inform the patient of the diagnosis and what the outcome of no treatment or the best conventional treatment is likely to be. Only against this background is it possible to obtain the informed consent and co-operation from the patient and family, without which comprehensive management cannot be undertaken. Thus, with even the established two-drug combinations, patients may experience many years of good quality life, particularly when attending a multidisciplinary clinic where all the various problems with the disease itself and the treatment can be anticipated and to a large extent circumvented. There does, however, come a time when relentless disease progression and failure to respond to drugs raise a question about persisting with therapy, particularly in the face of extensive skeletal disease damage, increasing age or failure of the patient or his family to give consent for extending treatment. Under the circumstances a more conservative approach may be necessary.

At the other extreme is the need to recognise that highly aggressive treatmen options may be urgently needed. Here, increasing doses of melphalan in combination with radiotherapy and then bone marrow transplantation exemplify current practice and will require some weeks in isolation, with the patient at risk from serious complications, including death. The appropriateness of this therapy depends upon the performance status of the patient and, to a very large extent, age. In the younger patient whose outlook for survival would be poor and who is, in any event, better likely to tolerate an aggressive management regimen, the likelihood of improved quality and quantity of life after the risks of the transplantation have passed is a compelling reason to follow this route, particularly in the light of steadily improving results.

\section{SUMMARY AND CONCLUSIONS}

Two comments are appropriate.

Firstly, there is the haematologic observation that quality and quantity of life can be improved by conventional treatment. The best results are obtained from a multidisciplinary health care group and considerable experience is necessary to select the option most likely to be of greatest benefit to any particular individual. However, even under these circumstances survival is modestly prolonged and modern treatment options must be appreciated as extending to biological immune response modulation and most particularly to the use of high doses of chemotherapy, often in combination with radiotherapy, followed by bone marrow transplantation.

The second point is equally important. This is that there is an integral part for the professional nurse to play in the multidisciplinary health care team. We no longer consider it appropriate for these colleagues to occupy any position other than an active role in all aspects of management. Most particularly is our viewpoint that the fulfillment of nursing ideals and academic aspirations must be recognised by establishing a specific career 
structure for specialists in this profession that corresponds to those existing within the medical hierarchy. Such a concept, well established in the United States of America, is that of the Clinical Nurse Specialist. Ideally, such individuals should have a demonstrated commitment to continuing with their education and will often be studying for higher university degrees. On this basis, over and above such broadly based studies, is their involvement in the design and operation of clinical protocols and participation in trials that range from therapeutic apheresis to the increasing use of growth factors, resulting in a degree of professionalism that guarantees their active participation in all aspects of patient management. This approach is also essential if precious nursing resources are to be retained within the health care system by providing a properly structured career for those who wish to continue actively practising their chosen profession. These general concepts have been demonstrated by highlighting the integral role played by the professional irse in optimally managing patients with myeloma.
Lucille Wood, R.N., R.M.

Chief Professional Nurse

*John D. M. Richards, M.D., F.R.C.P., F.R.C.Path.

Visiting Professor

Peter Jacobs, M.D., Ph.D.

Professor and Head from

the University of Cape Town Leukaemia Centre and Departments of Haematology. Groote Schuur Hospital, Cape Town and

* University College Hospital, London

Supported by the University of Cape Town Leukaemia Centre and Staff Research Fund, the Gwendoline Moore Trust, the National Cancer Association, the Medical Research Council and the Michael Chanani and Kaliski bequests.

Correspondence

Professor Peter Jacobs Department of Haematology

University of Cape Town Medical School Anzio Road, Observatory 7925

Cape, South Africa 